\title{
Higher satisfaction after total knee arthroplasty using restricted inverse kinematic alignment compared to adjusted mechanical alignment
}

\author{
Philip Winnock de Grave ${ }^{1}$ (D) $\cdot$ Thomas Luyckx $^{1,2} \cdot$ Kurt Claeys $^{3} \cdot$ Thomas Tampere $^{1,4} \cdot$ Jonas Kellens $^{1,2}$. \\ Jacobus Müller ${ }^{5} \cdot$ Paul Gunst ${ }^{1}$
}

Received: 25 May 2020 / Accepted: 14 July 2020 / Published online: 31 July 2020

(c) The Author(s) 2020

\begin{abstract}
Purpose Various alignment philosophies for total knee arthroplasty (TKA) have been described, all striving to achieve excellent long-term implant survival and good functional outcomes. In recent years, in search of higher functionality and patient satisfaction, a shift towards more tailored and patient-specific alignment is seen. The purpose of this study was to describe a restricted 'inverse kinematic alignment' (iKA) technique, and to compare clinical outcomes of patients that underwent robotic-assisted TKA performed by restricted iKA vs. adjusted mechanical alignment (aMA).

Methods The authors reviewed the records of a consecutive series of patients that received robotic-assisted TKA with restricted iKA $(n=40)$ and with aMA $(n=40)$. Oxford Knee Score (OKS) and satisfaction on a visual analogue scale (VAS) were collected at a follow-up of 12 months. Clinical outcomes were assessed according to patient acceptable symptom state (PASS) thresholds, and uni- and multivariable linear regression analyses were performed to determine associations of OKS and satisfaction with six variables (age, sex, body mass index (BMI), preoperative hip-knee-ankle (HKA) angle, preoperative OKS, alignment technique).

Results The restricted iKA and aMA techniques yielded comparable outcome scores $(p=0.069)$, with OKS, respectively, $44.6 \pm 3.5$ and $42.2 \pm 6.3$. VAS Satisfaction was better $(p=0.012)$ with restricted iKA $(9.2 \pm 0.8)$ compared to aMA $(8.5 \pm 1.3)$. The number of patients that achieved OKS and satisfaction PASS thresholds was significantly higher $(p=0.049$ and $p=0.003$, respectively) using restricted iKA (98\% and 80\%) compared to aMA (85\% and 48\%). Knees with preoperative varus deformity, achieved significantly $(p=0.025)$ better OKS using restricted iKA $(45.4 \pm 2.0)$ compared to aMA (41.4 \pm 6.8$)$. Multivariable analyses confirmed better OKS $(\beta=3.1 ; p=0.007)$ and satisfaction $(\beta=0.73 ; p=0.005)$ with restricted iKA.

Conclusions The results of this study suggest that restricted iKA and aMA grant comparable clinical outcomes at 12-month follow-up, though a greater proportion of knees operated by restricted iKA achieved the PASS thresholds for OKS and satisfaction. Notably. in knees with preoperative varus deformity, restricted iKA yielded significantly better OKS and satisfaction than aMA.
\end{abstract}

Level of evidence Level III, comparative study.

Keywords Arthroplasty · Knee replacement · Patient-specific alignment · Inverse kinematic alignment $\cdot$ Robotic surgical procedures $\cdot$ Patient-reported outcomes $\cdot$ Patient satisfaction

Philip Winnock de Grave

philip.winnockdegrave@azdelta.be

Extended author information available on the last page of the article

\section{Introduction}

In striving to improve patient satisfaction, alignment in total knee arthroplasty (TKA) is gaining increased attention in recent years. Various alignment philosophies have been described, and can be considered as three main categories [19]: (1) Systematic alignment, such as mechanical alignment (MA) [13] and anatomic alignment (AA) [12], aims for a strict neutral coronal alignment with a hip-knee-ankle 
(HKA) angle of $180^{\circ}$; (2) Patient-specific alignment, such as kinematic alignment (KA) [11], aims to maintain the native limb alignment; (3) Hybrid alignment, such as adjusted mechanical alignment (aMA) [25] and restricted kinematic alignment (rKA) [2], aim to maintain the native coronal alignment within a HKA angle safe zone of $177^{\circ}$ to $183^{\circ}$.

In a recent computed tomography (CT) study of 308 non-arthritic knees, Hirschmann et al. [10] estimated that native limb alignment in men and women, respectively, corresponds to MA in $4 \%$ and $6 \%$, to $\mathrm{AA}$ in $17 \%$ and $18 \%$, and to rKA in $45 \%$ and $51 \%$. Although patient-specific alignment techniques would maintain native alignment of all knees, safe ranges for postoperative residual varus or valgus remain unknown [2, 8-10] leading some surgeons to opt for hybrid alignment [18].

The KA technique aims to 'resurface' the femur maintaining the native femoral joint line obliquity, with the flexion and extension gaps balanced by adjusting the tibial resection. In some cases, KA involves complex algorithms to balance the flexion and extension gaps [19], which may result in more oblique tibial varus resections that sacrifice medial tibial bone stock. The first author introduced a new technique of restricted 'inverse kinematic alignment' (iKA) which aims to 'resurface' the tibia with equal medial and lateral resections maintaining the native tibial joint line obliquity, with the flexion and extension gaps balanced by adjusting the femoral resections. This technique could, therefore, avoid tibial overresection and tibia-related postoperative complications. The purpose of this study was to compare clinical outcomes of patients that underwent robotic-assisted TKA performed by restricted iKA vs. those with aMA. The null hypothesis was that both techniques would yield equivalent clinical scores and patient satisfaction. These findings could be relevant to the ongoing efforts, of improving clinical outcomes while restoring physiological joint line obliquity, using patientspecific alignment techniques.

\section{Materials and methods}

\section{Patients}

The authors reviewed the records of a consecutive series of patients that received robotic-assisted TKA (Stryker Triathlon ${ }^{\circledR}$ CR knee) with restricted iKA ( $n=50$, surgeon (1) and with aMA ( $n=50$, surgeon two). To account for the learning curve using robotic-assisted TKA, the first ten cases from each group were excluded (Fig. 1). The indication for surgery was end stage knee osteoarthritis of grade four according to Kellgren-Lawrence classification in at least one of the three knee compartments. Standard radiographic evaluation was carried out on weight-bearing radiographs: anteroposterior, Rosenberg, lateral, and skyline views.

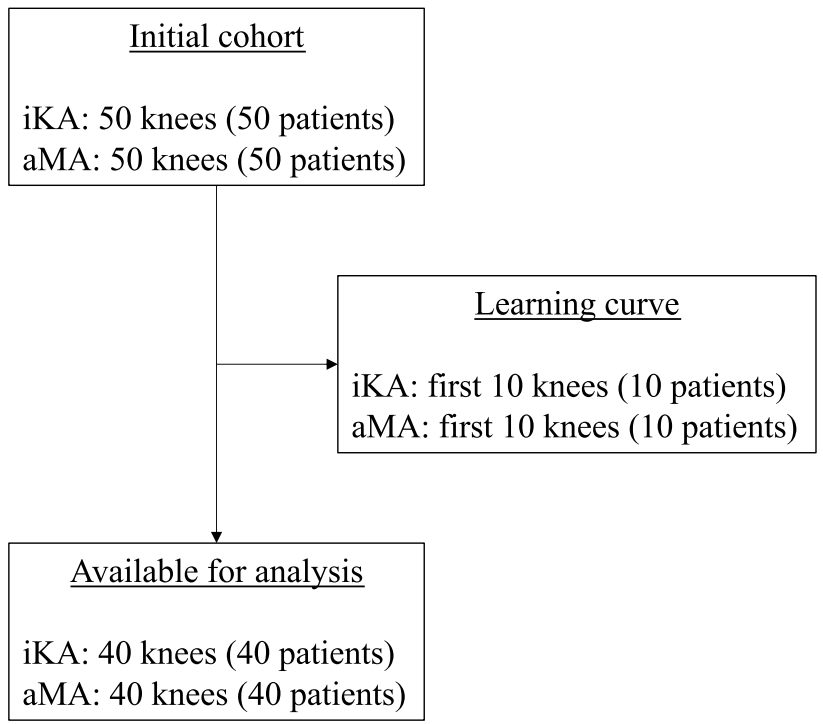

Fig. 1 Study flowchart

Clinical follow-up was organized at 6 weeks, 3 months and 1 year postoperative for both groups. All patients had provided written informed consent for the use of their data and images for research and publishing purposes, and the institutional review board approved the study, according the Helsinki guidelines.

\section{Surgical technique}

All patients underwent general anaesthesia with an additional ultrasound-guided, adductor canal sensory nerve block. For both restricted iKA and aMA groups, exposure was done by a far medial subvastus approach [22] with the medial joint capsule incised in two distinct layers. Arthrotomy was performed far medial just in front of the medial collateral ligament (MCL). When reaching the medial tibia, the longitudinal capsular incision (vertical) was redirected $90^{\circ}$ (horizontal) parallel to the tibial plateau in the lateral direction. No soft-tissues were peeled off from the anteromedial tibia. The knee was then brought into flexion and the tourniquet was deflated for the remainder of surgery.

The robotic system $\left(\mathrm{MAKO}^{\circledR}\right.$, Stryker, Kalamazoo, MI, USA) was set up and calibrated following the standard protocol [4]. A preoperative computed tomography (CT) scan of the hip, knee and ankle was uploaded to the TKA application platform for segmentation of a subject-specific three-dimensional model of the knee. During surgery, tibial and femoral trackers were fixed to the bones, and femur and tibia registration was performed by capturing 40 random points on the bony surface of each bone. As it is an image-based system that uses CT, bone registration was made with a sharp probe to guarantee bone contact in areas with cartilage or soft-tissue coverage. An accuracy level of less than $0.5 \mathrm{~mm}$ 
had to be achieved to be able to continue the procedure. All measurements were made intraoperatively using the robotic system user-interface, which has a resolution of $0.5 \mathrm{~mm}$ for distances and $0.1^{\circ}$ for angles.

The navigation tool of the robotic system and a manual ligament tensioning device was used to assess the overall alignment and the soft-tissue envelope both in extension and flexion, with the patella in place. The femoral and tibial components were virtually positioned according to the balancing principles of the alignment technique, i.e., restricted iKA or aMA (Fig. 2).

\section{iKA}

The tibial component was positioned first by planning a resection of equal amounts of bone medial and lateral on the tibia, taking into account bony wear. The aim was to restore the pre-arthritic medial proximal tibial angle (MPTA), within a safe zone of $84^{\circ}$ (varus) to $92^{\circ}$ (valgus), which represents native knee alignment in $93 \%$ of Caucasian knees [9]. The tibial slope was set equal to the native medial tibial slope. On the femoral side, the femoral component is positioned to restore the medial joint line height both in extension and flexion. The flexion and extension gaps are balanced by adjusting the distal lateral and posterior lateral resection levels on the femur. For the flexion gap, the aim was to achieve residual laxity of 1-2 $\mathrm{mm}$ in the medial compartment and 1-3 $\mathrm{mm}$ in the lateral compartment. For the extension gap, the aim was to achieve residual laxity of 1-2 $\mathrm{mm}$ in both compartments while remaining within an HKA angle safe zone of $174-183^{\circ}$, which accounts for native limb alignment in 96\% of Caucasian knees [8] (Fig. 3).

\section{aMA}

The adjusted Mechanical Alignment (aMA) technique is an adaptation of the conventional MA technique but with undercorrection of constitutional coronal deformity, within a limit of $\pm 3^{\circ}$. The femoral resection is adjusted to preserve mild constitutional deformity and/or reduce more severe deformity while leaving the tibial component mechanically aligned. The tibial component was positioned with the aim to be perpendicular $\left(90^{\circ}\right)$ to the mechanical tibial axis. The tibial slope was equal to the native medial tibial slope. For the flexion gap, the femoral component was positioned to achieve residual laxity of 1-2 $\mathrm{mm}$ in both compartments. Likewise, for the extension gap the femoral component was positioned to achieve a residual laxity of $1-2 \mathrm{~mm}$ in both compartments while remaining within an HKA angle safe zone of $177-183^{\circ}$.

\section{Resections}

Tibial and femoral resections were done according to the surgeon's defined intraoperative plan using the haptic robotic-assisted system. As a CT-based navigation tool is used, all planned resection thicknesses are resections of bone, without taking into account the cartilage. The robotic system allowed the surgeon to move the oscillating saw in the defined cutting plane within haptic boundaries, protecting the soft-tissues [4]. The patella was routinely resurfaced using a conventional oscillating saw.

\section{Rehabilitation}

Postoperative rehabilitation protocols included direct mobilization and immediate full weight bearing protected by crutches. With the guidance of a physiotherapist, patients were encouraged to perform exercises with active flexion and active extension movements from day 1. On average, patients stayed three nights in the hospital. Daily physiotherapy sessions were continued at home. The use of crutches was advised during the first 2 weeks. All patients received routine prophylaxis with low-molecular-weight heparin for 4 weeks after surgery. First postoperative appointment at the outpatient clinic was at 6 weeks.

\section{Clinical scores}

Oxford Knee Score (OKS) (worst, 0; best 48) was collected preoperatively and postoperatively at a follow-up of 12 months for all knees. Patients also indicated their satisfaction with the TKA on a Visual Analogue Scale (VAS) (worst, 0 ; best, 10). Clinical outcomes were assessed according to patient acceptable symptom state (PASS) thresholds. PASS is an absolute threshold proposed for symptomatic variables in osteoarthritis to determine the point beyond which patients consider themselves well and, as such, are satisfied with treatment. Recognized PASS thresholds are 37 for OKS [21] for OKS, and eight for VAS Satisfaction [3].

\section{Statistical analyses}

Descriptive statistics were used to summarize the data. Shapiro-Wilk tests were used to assess the normality of distributions. Differences in means between restricted iKA and aMA knees were, respectively, tested using the student $t$ test or analysis of variance, whereas associations between categorical variables were tested using the Kruskal-Wallis test. Based on the classification of Hirschmann et al. [9], which distinguishes five tibial phenotypes, the present study considered only three tibial phenotypes (varus, MPTA $<85^{\circ}$; neutral, $85^{\circ} \leq \mathrm{MPTA} \leq 89^{\circ}$; valgus, MPTA $>89^{\circ}$ ). This simplification was achieved by not considering different extents 


\section{iKA}
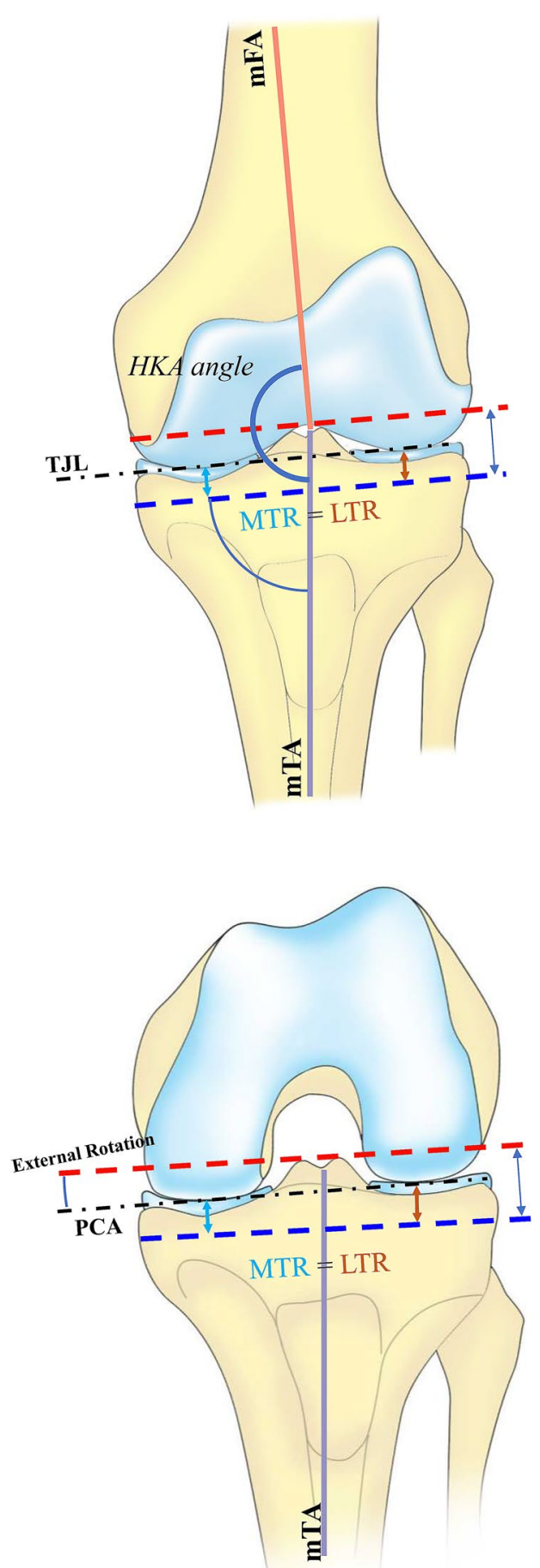

aMA

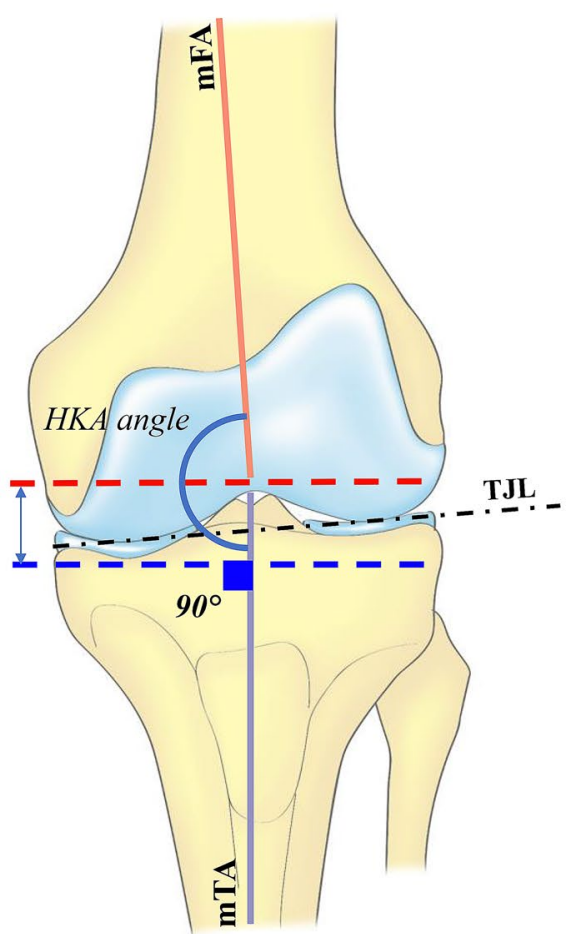

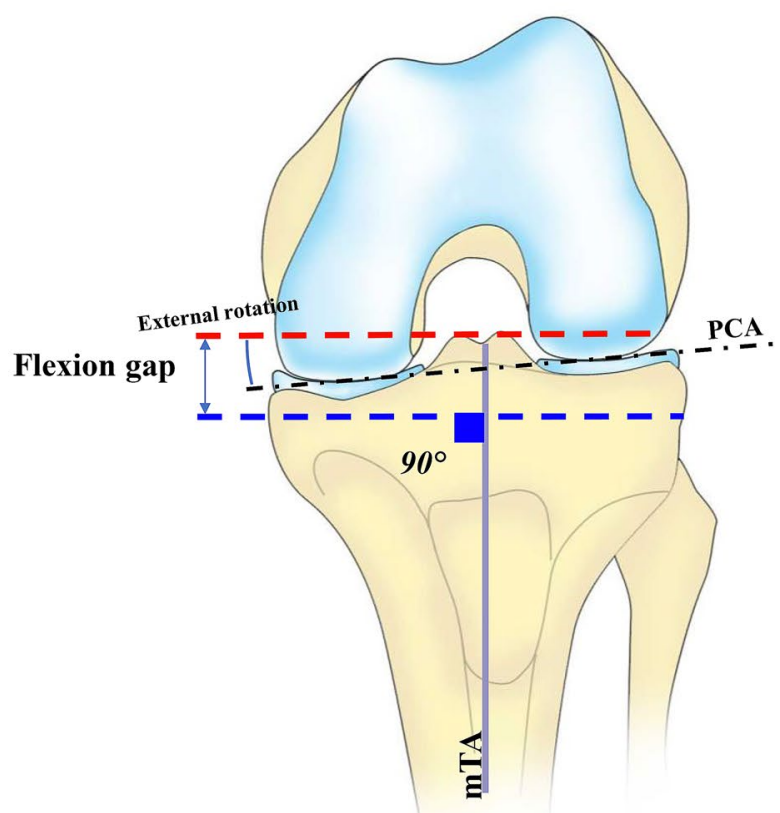

Fig. 2 Illustration of the iKA and aMA philosophy in a common knee, with an MPTA of $87^{\circ}$. * iKA technique: Tibial resection: tibial resection parallel to tibial joint line $T J L$, medial tibial resection $(M T R)=$ lateral tibial resection $(L T R)$. Hip-knee-ankle $(H K A)$ angle: restored to pre-arthritic HKA angle by tensioning soft-tissue envelope in extension and performing distal femoral resection. Extension gap laxity: 1-2 mm opening. Flexion gap: femoral rotation governed by soft-tissue tension in flexion with patella-in-place; $1-2 \mathrm{~mm}$ medial

and 1-3 mm lateral residual laxity.*aMA technique: Tibial resection: $90^{\circ}$ with the mechanical tibial axis $m T A$. HKA angle: restored to pre-arthritic HKA angle by soft-tissue envelope in extension and performing distal femoral resection, but within a HKA angle safe zone of 177-183. Extension gap laxity: 1-2 mm opening. Flexion gap: femoral rotation governed by soft-tissue tension in flexion with patella-inplace; $1-2 \mathrm{~mm}$ residual laxity medial and lateral. Abbreviations: $m T A$ mechanical tibial axis; $m F A$ mechanical femoral axis 
of varus and valgus alignment, and by defining the thresholds as $\pm 2^{\circ}$ instead of $\pm 1.5^{\circ}$. Femoral phenotypes were categorised according to their mechanical lateral distal femoral angles (mLDFA) using the classification of Yazdi et al. [26] (varus, mLDFA $>89^{\circ}$; neutral, $85^{\circ} \leq \mathrm{mLDFA} \leq 89^{\circ}$; valgus, $\mathrm{mLDFA}<85^{\circ}$ ). Preoperative radiographs were assessed according the Kellgren-Lawrence classification by two investigators involved in this study. Interobserver agreement was calculated using intraclass correlation coefficients (ICC) which can be interpreted as follows [5]: $<0.40$ poor; 0.40-0.59 fair; 0.60-0.74 good; 0.75-1.00 excellent. The differences between the pre-, postoperative and net change in OKS, and postoperative VAS Satisfaction in the restricted iKA and aMA groups were analysed in three ways:
(1) The difference in means between the two groups; (2) the difference in means between the two groups with knees categorised according to preoperative deformity, i.e., varus $\left(\right.$ HKA angle $\left.<177^{\circ}\right)$, neutral $\left(\right.$ HKA angle $\left.=177-183^{\circ}\right)$ and valgus (HKA angle $>183^{\circ}$ ); and (3) the difference in proportions (\%) of knees that were above the PASS thresholds. Considering the findings of Dossett et al. [6] who reported OKS $(40 \pm 10.2)$ in knees after KA TKA, and to determine whether a difference of five points in OKS is statistically significant, a priori sample size calculation indicated that a minimum of 39 knees per group was necessary to achieve a power of $70 \%$ (G*Power 3.1, Heinrich-Heine-Universität Düsseldorf, Germany). To ascertain detection of the effects of alignment technique on OKS, the sample size used for the $\mathbf{a}$

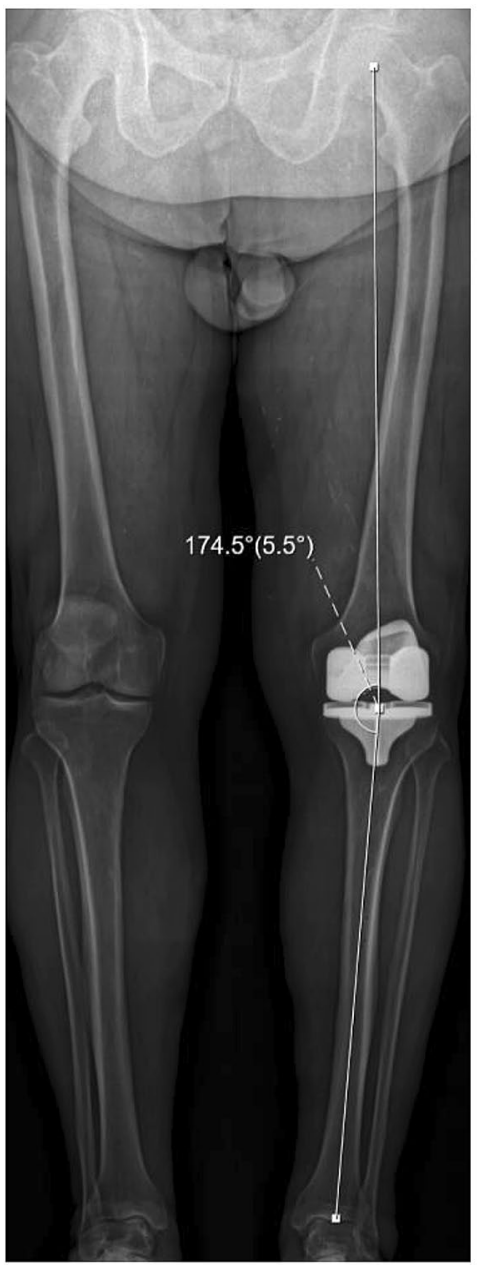

b

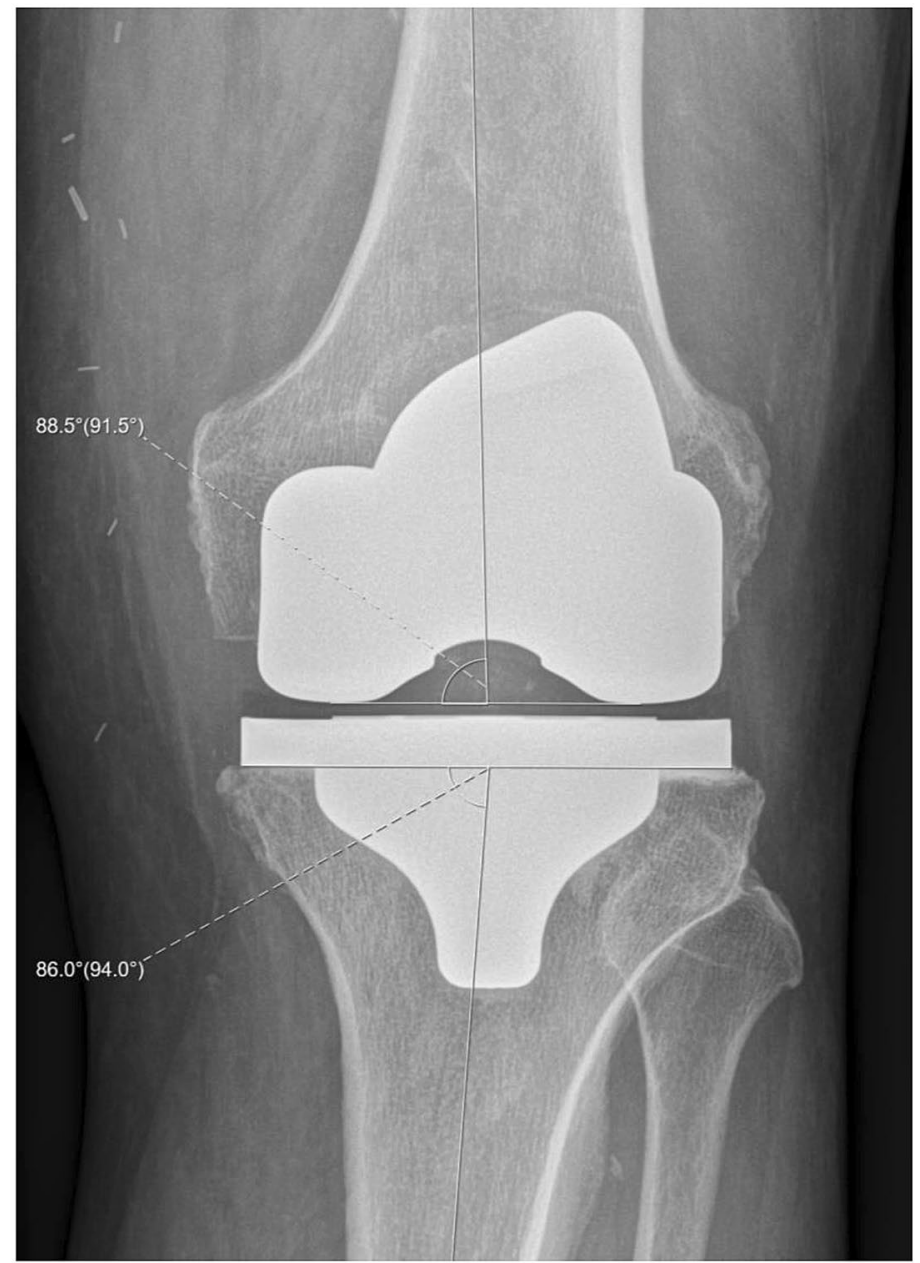

Fig. 3 Postoperative radiographs of 76 years, male patient who received a left TKA by iKA. The patient suffered invalidating knee pain caused by tricompartmental osteoarthritis and progressive varus deformity with obliteration of the medial joint space (grade 4). a
Postoperative standing full-leg X-ray (EOS) showing a restored HKA angle of $174,5^{\circ}$ and bilateral symmetrical joint line obliquity, parallel to the floor. b Postoperative weight-bearing X-ray of the knee detailing a restored MPTA of $86^{\circ}$ incombination with an mLDFA of $91,5^{\circ}$ 
Table 1 Patient demographics

\begin{tabular}{|c|c|c|c|c|c|}
\hline & \multicolumn{2}{|l|}{$\mathrm{iKA}(n=40)$} & \multicolumn{2}{|l|}{ aMA $(n=40)$} & \multirow[t]{2}{*}{$p$ value } \\
\hline & Mean \pm SD $(\%)$ & (range) & Mean \pm SD $(\%)$ & (range) & \\
\hline Age (years) & $69.9 \pm 8.3$ & $(54-86)$ & $67.4 \pm 9.5$ & $(50-89)$ & $n . s$ \\
\hline BMI $\left(\mathrm{kg} / \mathrm{m}^{2}\right)$ & $29.2 \pm 4.8$ & $(21.4-42.6)$ & $30.0 \pm 5.3$ & $(21.3-45.1)$ & $n . s$ \\
\hline Women & $25(60 \%)$ & & $23(58 \%)$ & & $n . s$ \\
\hline \multicolumn{6}{|l|}{ Preoperative HKA angle } \\
\hline Varus knees $\left(<177^{\circ}\right)$ & $23(58 \%)$ & & $21(53 \%)$ & & n.s \\
\hline Neutral knees $\left(177-183^{\circ}\right)$ & $13(33 \%)$ & & $15(38 \%)$ & & $n . s$ \\
\hline Valgus knees $\left(>183^{\circ}\right)$ & $4(10 \%)$ & & $4(10 \%)$ & & $n . s$ \\
\hline \multicolumn{6}{|l|}{ Preoperative MPTA } \\
\hline Varus knees $\left(<85^{\circ}\right)$ & $1(3 \%)$ & & $4(10 \%)$ & & $n . s$ \\
\hline Neutral knees $\left(85-89^{\circ}\right)$ & $38(95 \%)$ & & $31(78 \%)$ & & $n . s$ \\
\hline Valgus knees $\left(>89^{\circ}\right)$ & $1(3 \%)$ & & $5(13 \%)$ & & $n . s$ \\
\hline \multicolumn{6}{|l|}{ Preoperative mLDFA } \\
\hline Varus knees $\left(>89^{\circ}\right)$ & $8(20 \%)$ & & $5(13 \%)$ & & $n . s$ \\
\hline Neutral knees $\left(85-89^{\circ}\right)$ & $32(80 \%)$ & & $35(88 \%)$ & & $n . s$ \\
\hline Valgus knees $\left(<85^{\circ}\right)$ & $0(0 \%)$ & & $0(0 \%)$ & & \\
\hline \multicolumn{6}{|c|}{ Kellgren-Lawrence classification } \\
\hline Medial compartment & & & & & n.s \\
\hline$\leq 2$ & 6 & & 8 & & \\
\hline 3 & 9 & & 11 & & \\
\hline 4 & 25 & & 21 & & \\
\hline Lateral compartment & & & & & n.s \\
\hline$\leq 2$ & 21 & & 19 & & \\
\hline 3 & 10 & & 11 & & \\
\hline 4 & 9 & & 10 & & \\
\hline Patellofemoral compartment & & & & & n.s \\
\hline$\leq 2$ & 8 & & 3 & & \\
\hline 3 & 13 & & 17 & & \\
\hline 4 & 19 & & 20 & & \\
\hline
\end{tabular}

$i K A$ inverse kinematic alignment; $a M A$ adjusted mechanical alignment; $S D$ standard deviation; deg, degrees; BMI, body mass index; HKA, Hip-Knee-Ankle; MPTA medial proximal tibial angle, $m L D F A$ mechanical lateral distal femoral angle

present study was adequate, 80 knees with 40 in each group. Uni- and multivariable linear regression analyses were performed to determine associations of three outcomes (Postoperative OKS, OKS net change, VAS satisfaction) and six variables (age, sex, BMI, preoperative HKA angle, preoperative $\mathrm{OKS}$, alignment technique). Multivariable regression models were deemed sufficiently powered, considering the recommendations of Austin and Steyerberg [4] of ten Subject Per Variable (SPV). A $p$ value $<0.05$ was considered statistically significant. Statistical analyses were performed using R version 3.3.2 (R Foundation for Statistical Computing, Vienna, Austria).

\section{Results}

\section{Preoperative demographics}

The mean age and BMI, and distributions of sex, preoperative HKA angle, MPTA, mLDFA and Kellgren-Lawrence classification were the same between the 2 groups (Table 1). Preoperative HKA angles revealed that most knees were in varus (HKA angle $<177^{\circ}$; restricted $\mathrm{iKA}, 173.3^{\circ} \pm 1.9^{\circ}$ $(n=23)$ vs. aMA, $\left.173.4^{\circ} \pm 2.2^{\circ}(n=21)\right)$, followed by neutral $\left(\mathrm{HKA}\right.$ angle $=177-183^{\circ}$; restricted $\mathrm{iKA}, 178.7^{\circ} \pm 2.2^{\circ}$ 
Table 2 Intraoperative measurements, settings and clinical scores

\begin{tabular}{|c|c|c|c|}
\hline & $\begin{array}{l}\mathrm{iKA}(n=40) \\
\text { Mean } \pm \text { SD }\end{array}$ & $\begin{array}{l}\text { aMA }(n=40) \\
\text { Mean } \pm \text { SD }\end{array}$ & $p$ value \\
\hline \multicolumn{4}{|l|}{ Angles } \\
\hline \multicolumn{4}{|l|}{ HKA angle } \\
\hline Preoperative (deg) & $176.3 \pm 4.3$ & $176.9 \pm 4.6$ & $n . s$ \\
\hline Postoperative (deg) & $178.3 \pm 2.1$ & $179.6 \pm 1.9$ & 0.003 \\
\hline Net change (deg) & $2.0 \pm 2.8$ & $2.7 \pm 3.2$ & $n . s$ \\
\hline \multicolumn{4}{|l|}{ MPTA } \\
\hline Preoperative (deg) & $86.7 \pm 1.3$ & $87.4 \pm 1.7$ & 0.021 \\
\hline Postoperative (deg) & $87.1 \pm 1.4$ & $89.6 \pm 0.9$ & $<0.001$ \\
\hline Net change (deg) & $0.4 \pm 0.7$ & $2.2 \pm 1.5$ & $<0.001$ \\
\hline \multicolumn{4}{|l|}{ mLDFA } \\
\hline Preoperative (deg) & $88.0 \pm 1.4$ & $87.7 \pm 1.4$ & $n . s$ \\
\hline Postoperative (deg) & $88.8 \pm 1.4$ & $90.0 \pm 1.6$ & $<0.001$ \\
\hline Net change (deg) & $0.9 \pm 1.2$ & $2.3 \pm 1.0$ & $<0.001$ \\
\hline Femoral rotation from PCA (deg) & $2.3 \pm 1.4$ & $4.8 \pm 2.3$ & $<0.001$ \\
\hline Femoral rotation from TEA (deg) & $-0.7 \pm 2.1$ & $2.2 \pm 2.5$ & $<0.001$ \\
\hline Tibial Slope (deg) & $4.2 \pm 1.2$ & $4.1 \pm 1.6$ & $n . s$ \\
\hline \multicolumn{4}{|l|}{ Resections } \\
\hline \multicolumn{4}{|l|}{ Tibial resection } \\
\hline Medial (mm) & $5.4 \pm 0.9$ & $4.4 \pm 1.2$ & $<0.001$ \\
\hline Lateral (mm) & $6.1 \pm 1.2$ & $6.2 \pm 1.3$ & $n . s$ \\
\hline \multicolumn{4}{|l|}{ Femoral resection } \\
\hline Distal medial $(\mathrm{mm})$ & $6.4 \pm 0.8$ & $7.1 \pm 1.4$ & 0.021 \\
\hline Distal lateral (mm) & $4.7 \pm 1.3$ & $3.4 \pm 1.6$ & $<0.001$ \\
\hline Posterior medial (mm) & $8.2 \pm 1.2$ & $9.4 \pm 1.4$ & $<0.001$ \\
\hline Posterior lateral (mm) & $6.6 \pm 1.0$ & $5.7 \pm 2.0$ & 0.014 \\
\hline \multicolumn{4}{|l|}{ Clinical scores } \\
\hline \multicolumn{4}{|l|}{ OKS (worst, 0 ; best, 48) } \\
\hline Preoperative & $26.3 \pm 6.4$ & $27.2 \pm 5.2$ & $n . s$ \\
\hline Postoperative & $44.8 \pm 3.5$ & $42.2 \pm 6.3$ & n.s \\
\hline Net change & $18.6 \pm 7.0$ & $15.0 \pm 8.6$ & $n . s$ \\
\hline VAS Satisfaction (worst, 0; best, 10) & $9.2 \pm 0.8$ & $8.5 \pm 1.3$ & 0.012 \\
\hline \multicolumn{4}{|l|}{ PASS achieved } \\
\hline OKS & $39(98 \%)$ & $34(85 \%)$ & 0.049 \\
\hline VAS satisfaction & $32(80 \%)$ & $19(48 \%)$ & 0.003 \\
\hline
\end{tabular}

$i K A$, inverse kinematic alignment; $a M A$ adjusted mechanical alignment; $S D$ standard deviation; deg degrees; HKA Hip-Knee-Ankle; MPTA medial proximal tibial angle; $m L D F A$ mechanical lateral distal femoral angle; $P C A$ posterior condylar axis; TEA transepicondylar axis; $O K S$ Oxford Knee Score; VAS visual analog scale; $P A S S$ patient acceptable symptom state $(n=13)$ vs. aMA, $\left.179.7^{\circ} \pm 2.3^{\circ}(n=15)\right)$ and only a few in valgus (HKA angle $>183^{\circ}$; restricted $\mathrm{iKA}, 185.0^{\circ} \pm 0.8^{\circ}$ $(n=4)$ vs. aMA, $\left.185^{\circ} \pm 0.8^{\circ}(n=4)\right)$. Interobserver agreement on Kellgren-Lawrence classifications was excellent $(\mathrm{ICC}>0.9)$.

\section{Overall outcomes}

The difference in preoperative MPTA between the two groups was statistically significant $(p=0.021)$, but clinically not meaningful, since the difference was $<1^{\circ}$ (Table 2 ). The postoperative MPTA and HKA angles were significantly ( $p<0.001$ and $p=0.003$, respectively) more in varus in the restricted iKA group $\left(87.1^{\circ} \pm 1.4^{\circ}\right.$ and $178.3^{\circ} \pm 2.1^{\circ}$, respectively), compared with the aMA group $\left(89.6^{\circ} \pm 0.9^{\circ}\right.$ and $179^{\circ} \pm 1.9^{\circ}$, respectively). Femoral components were significantly $(p<0.001)$ less externally rotated relative to the native PCA in the restricted iKA group $\left(2.3^{\circ} \pm 1.4^{\circ}\right)$, compared with the aMA group $\left(4.8^{\circ} \pm 2.3^{\circ}\right)$. Medial tibial resections were significantly $(p<0.001)$ deeper in the 


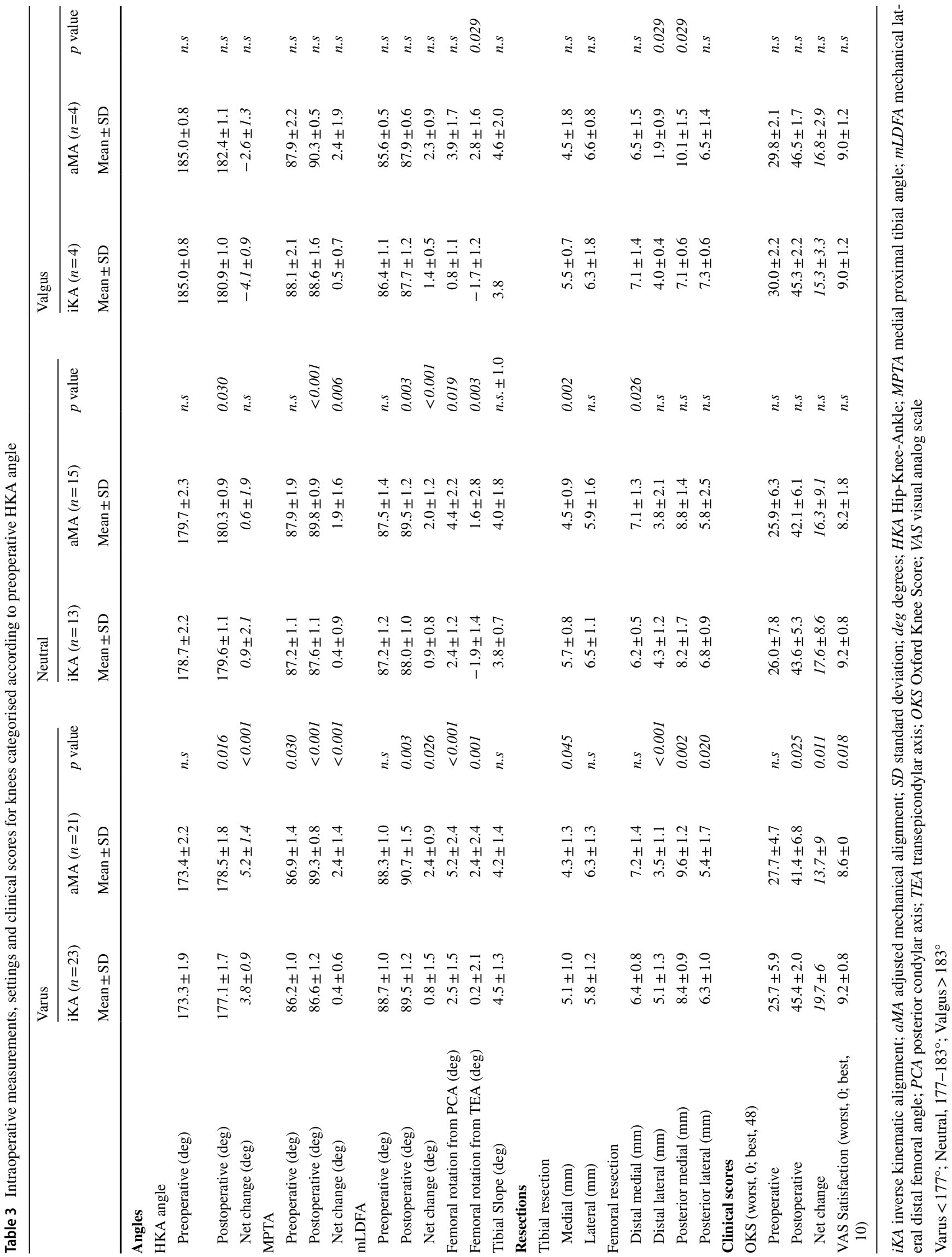


restricted iKA group $(5.4 \pm 0.9 \mathrm{~mm})$, compared to the aMA group $(4.4 \pm 1.2 \mathrm{~mm})$.

No complications occurred during surgery. Knee stability and patellar tracking were assessed with no necessity of soft-tissue and lateral releases in both groups. In the aMA group, one patient received a mobilisation under analgesia at 2 months postoperatively. None of the knees were revised or being considered for revision at the latest follow-up.

There were no significant differences in preoperative OKS, postoperative OKS or the net change in OKS between the two groups at 1-year follow-up. VAS satisfaction was significantly $(p=0.012)$ higher in the restricted iKA group $(9.2 \pm 0.8)$ compared with the aMA group $(8.5 \pm 1.3)$. There were significantly $(p=0.049)$ more knees that exceeded the OKS PASS threshold with restricted iKA [39 (98\%)], compared with aMA [34 (85\%)]. Likewise, significantly ( $p=0.003)$ more knees exceeded the satisfaction PASS threshold using restricted iKA [32 (80\%)] compared to using aMA [19 (48\%)].

\section{Effect of preoperative deformity}

The alignment, resections and clinical outcomes with restricted iKA and aMA on varus $\left(\mathrm{HKA}<177^{\circ}\right)$, neutral $\left(\mathrm{HKA}=177-183^{\circ}\right)$ and valgus $\left(\mathrm{HKA}>183^{\circ}\right)$ knees are shown in (Table 3 ). The net change in HKA angle was significantly $(p<0.001)$ less for varus knees in the restricted iKA group $\left(3.8^{\circ} \pm 0.9^{\circ}\right)$, compared with the aMA group $\left(5.2^{\circ} \pm 1.4^{\circ}\right)$. There were no significant differences in postoperative MPTA and HKA of valgus knees between the two groups. Postoperative and net improvement of OKS in varus knees was significantly ( $p=0.025$ and $p=0.011$, respectively) higher in the restricted iKA group ( $45.4 \pm 2.0$ and $19.7 \pm 6.4$, respectively) compared with the aMA group (41.4 \pm 6.8 and $13.7 \pm 9.1$, respectively). Finally, the VAS satisfaction in varus knees was significantly $(p=0.018)$ higher in the restricted iKA group $(9.2 \pm 0.8)$ compared with the aMA group $(8.6 \pm 0.9)$.

\section{Regression analysis}

Univariable analysis revealed decreasing postoperative OKS with age $(\beta=-0.1 ; p=0.047)$ and better OKS with restricted iKA $(\beta=2.6 ; p=0.023)$. Multivariable analysis confirmed better postoperative OKS with restricted iKA $(\beta=3.1 ; p=0.007)$, but also worse OKS for women $(\beta=-2.4 ; p=0.045)$ (Table 4). Univariable analysis revealed less improvement in OKS with age $(\beta=-0.2 ; p=0.049)$ and preoperative OKS $(\beta=-1.0 ; p<0.001)$, but better improvement with restricted iKA $(\beta=3.6 ; p=0.043)$. Multivariable analysis confirmed less improvement in OKS with higher preoperative OKS $(\beta=-1.1 ; p<0.001)$, and better improvement with restricted iKA $(\beta=3.1 ; p=0.007)$, but also less improvement for women $(\beta=-2.4 ; p=0.045)$. Univariable analysis revealed better VAS satisfaction with restricted iKA $(\beta=0.70 ; p=0.005)$, which was confirmed with multivariable analysis $(\beta=0.73$; $p=0.005)$.

\section{Discussion}

The most important finding of the present study was that robotic-assisted TKA performed by restricted iKA and aMA granted comparable clinical outcome, regarding OKS at 12-month follow-up. However, multivariable analyses revealed significantly better postoperative and net improvement in OKS as well as satisfaction for restricted iKA, compared to aMA. Finally, in knees with preoperative varus deformity, restricted iKA yielded significantly better OKS and satisfaction. These findings, therefore, partly refute the null hypothesis.

The clinical outcomes of the restricted iKA and aMA techniques in the present study were equivalent and, in some cases, better than findings of other studies that compared TKA performed by KA and MA. In a randomised control trial (RCT), Young et al. [6] found no significant difference between KA $(n=49)$ and MA $(n=50)$ in OKS $(42 \pm 6$ and $41 \pm 6$, respectively) at 24-month follow-up. Conversely, an RCT by Dossett et al. [27] revealed a significant $(p=0.005)$ difference between KA $(n=44)$ and MA $(n=44)$ in OKS $(40 \pm 10.2$ and $33 \pm 11.1$, respectively) at 24-month followup. Interestingly, $90 \%$ of knees in the latter study were predisposed to varus alignment, for whom KA resulted in better OKS scores. In the present study, restricted iKA resulted in significantly better OKS compared to aMA for knees with varus deformity.

The restricted iKA technique yielded significantly greater proportions of knees (98\%) that reached the OKS patient acceptable symptom state (PASS) threshold, compared to aMA (85\%). By definition, the MA technique modifies the joint line obliquity in most cases, unlike patient-specific techniques, such as KA and restricted iKA. Interestingly, Nakajima et al. [16] found better functional outcomes in knees with postoperative joint line obliquity $\geq 2^{\circ}$ compared to knees with postoperative joint line obliquity $<2^{\circ}$. Moreover, Thienpont et al. [24] revealed that in varus knees the joint line convergence angle (the difference between the tibial joint line and the femoral joint line) is $3^{\circ} \pm 2^{\circ}$. The restricted iKA technique restores the joint line obliquity of the tibia, whereas KA restores the joint line obliquity of the femur. Therefore, in varus knees, the postoperative joint line in restricted iKA will be $3^{\circ} \pm 2^{\circ}$ less oblique, compared to KA.

Valgus knees have been shown to have hypoplastic lateral femoral condyles $\left(\mathrm{mLDFA}\right.$ of $85^{\circ} \pm 2.3^{\circ}$ ) [15] with neutral tibiae (MPTA of $90^{\circ} \pm 1.5^{\circ}$ ) [10]. KA aims to resurface the 


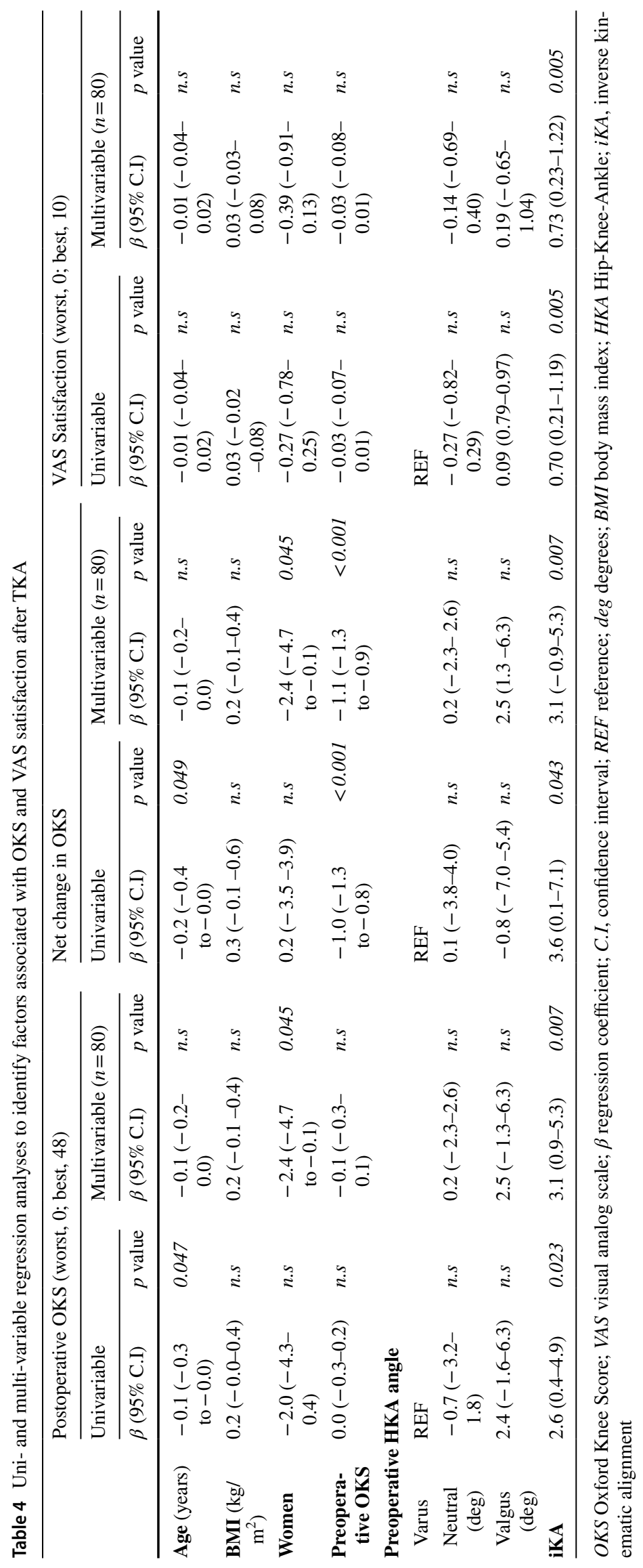


femur, which might result in oblique valgus femoral resections. Subsequent balancing on the tibia might require an oblique varus resection on a neutral tibia, which will sacrifice important medial tibial bone stock. Since the restricted iKA technique aims to resurface the tibia, the tibial resection will be neutral to the tibial mechanical axis, with equal medial and lateral tibial resections, thereby avoiding tibial over-resection and possibly tibia-related complications. In addition, multiplanar hypoplasia of the lateral femoral condyle in valgus knees is a risk factor for patellar instability [7], and there is a correlation between valgus alignment, femoral rotation and trochlear dysplasia [20]. Thienpont et al. [23] suggested that valgus knees, therefore, require external rotation to restore the native trochlear groove. In contrast to KA, restricted iKA allows for external rotation of the femoral component, which could favour functional outcomes in valgus knees.

This study has a number of limitations which should be acknowledged. First, restricted iKA was used by one surgeon, and aMA was used by another surgeon, which could introduce bias. However, both are experienced, high-volume surgeons ( $>100$ robotic-assisted TKAs annually each), where the surgical approach, robotic assistance, TKA implant and postoperative rehabilitation were identical. Second, both restricted iKA and aMA were performed with robotic assistance, and the findings may not apply to TKA cohorts using conventional instruments. Nevertheless, restricted iKA relies on a 'tibiafirst' approach, which most surgeons are familiar with, and which could be performed with conventional extramedullary tibial cutting-guides. Third, this was a retrospective study with only 12-month follow-up, and longer term outcomes at 24 and 60 months are still to be confirmed. Finally, preoperative MPTA and mLDFA phenotype classifications were not included in the multivariable analyses due to the small sample size, but distributions of preoperative osteoarthritic phenotypes between the two groups were equal.

This comparative study is the first describing the restricted iKA technique and reporting on functional outcomes. The clinical relevance of these findings is that the new technique of restricted iKA, with more physiological joint line obliquity, is a promising alignment strategy that merits further investigation and comparison to other patientspecific alignment techniques.

\section{Conclusion}

The results of this study suggest that restricted iKA and aMA grant comparable clinical outcomes at 12-month follow-up, though a greater proportion of knees operated by restricted iKA achieved the PASS thresholds for OKS and satisfaction. Notably, in knees with preoperative varus deformity, restricted iKA yielded significantly better OKS and satisfaction than aMA.
Author contributions PWDG: study design, recruiting, data collection, literature review, data interpretation, manuscript writing. TL: manuscript editing. KC: manuscript editing. TT: manuscript editing. JK: data collection. JM: statistical analysis. PG: recruiting, manuscript editing.

Funding There is no funding source.

\section{Compliance with ethical standard}

Conflict of interest The authors declare that they have no competing interests.

Ethical approval The institutional review board approved the study, according the Helsinki guidelines. (IRB reference number: B117201942336).

Informed consent All patients had provided written informed consent for the use of their data and images for research and publishing purposes.

Open Access This article is licensed under a Creative Commons Attribution 4.0 International License, which permits use, sharing, adaptation, distribution and reproduction in any medium or format, as long as you give appropriate credit to the original author(s) and the source, provide a link to the Creative Commons licence, and indicate if changes were made. The images or other third party material in this article are included in the article's Creative Commons licence, unless indicated otherwise in a credit line to the material. If material is not included in the article's Creative Commons licence and your intended use is not permitted by statutory regulation or exceeds the permitted use, you will need to obtain permission directly from the copyright holder. To view a copy of this licence, visit http://creativecommons.org/licenses/by/4.0/.

\section{References}

1. Allen MM, Pagnano MW (2016) Neutral mechanical alignment: is it necessary? Bone Joint J 98(1):81-83

2. Almaawi AM, Hutt JRB, Masse V, Lavigne M, Vendittoli PA (2017) The impact of mechanical and restricted kinematic alignment on knee anatomy in total knee arthroplasty. J Arthroplasty 32(7):2133-2140

3. Beck EC, Nwachukwu BU, Mehta N, Jan K, Okoroha KR, Rasio J, Nho SJ (2020) Defining meaningful functional improvement on the visual analog scale for satisfaction at 2 years after hip arthroscopy for femoroacetabular impingement syndrome. Arthroscopy 36(3):734-742.e732

4. Calliess T, Ettinger M, Savov P, Karkosch R, Windhagen $H$ (2018) Individualized alignment in total knee arthroplasty using image-based robotic assistance : Video article. Orthopade 47(10):871-879

5. Cicchetti D (1994) Guidelines, criteria, and rules of thumb for evaluating normed and standardized assessment instruments in psychology. Psychol Assess 6(4):284-290

6. Dossett HG, Estrada NA, Swartz GJ, LeFevre GW, Kwasman BG (2014) A randomised controlled trial of kinematically and mechanically aligned total knee replacements. Bone Joint J 96(7):907-913

7. Gillespie D, Mandziak D, Howie C (2015) Influence of posterior lateral femoral condyle geometry on patellar dislocation. Arch Orthop Trauma Surg 135(11):1503-1509 
8. Hirschmann MT, Hess S, Behrend H, Amsler F, Leclercq V, Moser LB (2019) Phenotyping of hip-knee-ankle angle in young non-osteoarthritic knees provides better understanding of native alignment variability. Knee Surg Sports Traumatol Arthrosc 27(5):1378-1384

9. Hirschmann MT, Moser LB, Amsler F, Behrend H, Leclercq V, Hess S (2019) Phenotyping the knee in young non-osteoarthritic knees shows a wide distribution of femoral and tibial coronal alignment. Knee Surg Sports Traumatol Arthrosc 27(5):1385-1393

10. Hirschmann MT, Moser LB, Amsler F, Behrend H, Leclerq V, Hess S (2019) Functional knee phenotypes: a novel classification for phenotyping the coronal lower limb alignment based on the native alignment in young non-osteoarthritic patients. Knee Surg Sports Traumatol Arthrosc 27(5):1394-1402

11. Howell SM, Hull ML (2012) Kinematic alignment in total knee arthroplasty. Elsevier, Philadelphia, PA

12. Hungerford DS, Kenna RV, Krackow KA (1982) The porouscoated anatomic total knee. Orthop Clin North Am 13(1):103-122

13. Insall JN, Binazzi R, Soudry M, Mestriner LA (1985) Total knee arthroplasty. Clin Orthop Relat Res NO 192:13-22

14. Liu HX, Shang P, Ying XZ, Zhang Y (2016) Shorter survival rate in varus-aligned knees after total knee arthroplasty. Knee Surg Sports Traumatol Arthrosc 24(8):2663-2671

15. Matsuda S, Miura H, Nagamine R, Mawatari T, Tokunaga M, Nabeyama R, Iwamoto Y (2004) Anatomical analysis of the femoral condyle in normal and osteoarthritic knees. J Orthop Res 22(1):104-109

16. Nakajima A, Sonobe M, Akatsu Y, Aoki Y, Takahashi H, Suguro T, Nakagawa K (2018) Association between limb alignment and patient-reported outcomes after total knee arthroplasty using an implant that reproduces anatomical geometry. J Orthop Surg Res 13(1):320

17. Nedopil AJ, Howell SM, Hull ML (2017) What mechanisms are associated with tibial component failure after kinematicallyaligned total knee arthroplasty? Int Orthop 41(8):1561-1569

18. Rivière C, Iranpour F, Auvinet E, Howell S, Vendittoli P-A, Cobb J, Parratte S (2017) Alignment options for total knee arthroplasty: a systematic review. Orthop Traumatol Surg Res 103(7):1047-1056
19. Rivière C, Lazic S, Boughton O, Wiart Y, Villet L, Cobb J (2018) Current concepts for aligning knee implants: patient-specific or systematic? EFORT Open Rev 3(1):1-6

20. Roger J, Lustig S, Cerciello S, Bruno CF, Neyret P, Servien E (2019) Short lateral posterior condyle is associated with trochlea dysplasia and patellar dislocation. Knee Surg Sports Traumatol Arthrosc 27(3):731-739

21. Scholes C, Ebrahimi M, Ektas N, Ireland J (2019) Efficacy of a second-generation rotating bearing tibial platform in total knee arthroplasty: a prospective observational cohort study with registry analysis. J Knee Surg. https://doi.org/10.1055/s-0039-16786 79

22. Thienpont E (2013) Faster quadriceps recovery with the far medial subvastus approach in minimally invasive total knee arthroplasty. Knee Surg Sports Traumatol Arthrosc 21(10):2370-2374

23. Thienpont E, Schwab PE, Paternostre F, Koch P (2014) Rotational alignment of the distal femur: anthropometric measurements with CT-based patient-specific instruments planning show high variability of the posterior condylar angle. Knee Surg Sports Traumatol Arthrosc 22(12):2995-3002

24. Thienpont E, Schwab PE, Cornu O, Bellemans J, Victor J (2017) Bone morphotypes of the varus and valgus knee. Arch Orthop Trauma Surg 137(3):393-400

25. Vanlommel L, Vanlommel J, Claes S, Bellemans J (2013) Slight undercorrection following total knee arthroplasty results in superior clinical outcomes in varus knees. Knee Surg Sports Traumatol Arthrosc 21(10):2325-2330

26. Yazdi H, Nazarian A, Wu JS, Amiri A, Hafezi P, Babikian M, Mohamadi A, Pakdaman R, Ghorbanhoseini M (2018) Different references for valgus cut angle in total knee arthroplasty. Arch Bone Jt Surg 6(4):289-293

27. Young SW, Walker ML, Bayan A, Briant-Evans T, Pavlou P, Farrington B (2017) The Chitranjan S. Ranawat Award : no difference in 2-year functional outcomes using kinematic versus mechanical alignment in TKA: a randomized controlled clinical trial. Clin Orthop Relat Res 475(1):9-20

Publisher's Note Springer Nature remains neutral with regard to jurisdictional claims in published maps and institutional affiliations.

\section{Affiliations}

\section{Philip Winnock de Grave ${ }^{1}$ (D) Thomas Luyckx ${ }^{1,2} \cdot$ Kurt Claeys $^{3} \cdot$ Thomas Tampere $^{1,4} \cdot$ Jonas Kellens $^{1,2}$. Jacobus Müller ${ }^{5}$. Paul Gunst ${ }^{1}$}

1 Dept. Orthopaedic Surgery, AZ Delta Roeselare, Brugsesteenweg 90, 8800 Roeselare, Belgium

2 Dept. Orthopaedic Surgery, UZ Leuven, Herestraat 49, 3000 Leuven, Belgium

3 Dept. Rehabilitation Sciences, KU Leuven, Spoorwegstraat 12, 8200 Brugge, Belgium
4 Dept. Orthopaedic Surgery, UZ Gent, De Pintelaan 185, 9000 Gent, Belgium

5 ReSurg SA, Rue Saint-Jean 22, 1260 Nyon, Switzerland 